\title{
Maximizing Network Lifetime through Optimal Power Consumption in Wireless Sensor Networks
}

\author{
El Abdellaoui Saîd ${ }^{1}$, Fakhri Youssef ${ }^{1,2}$, Debbah Merouane ${ }^{3}$, and Aboutajdine Driss ${ }^{1}$ \\ ${ }^{1}$ LRIT, Unité Associée au CNRST (URAC 29), Faculty of Sciences, \\ University Mohammed V- Agdal, Rabat, Morocco \\ ${ }^{2}$ LARIT, équipe Réseaux et Télécommunication, Faculty of Sciences, \\ University Ibn Tofail, Kenitra, Morocco \\ ${ }^{3}$ Alcatel-Lucent Chair on Flexible Radio, Supelec, Gif-sur-Yvette Cedex, France \\ elabdellaoui.said@yahoo.fr, fakhri-youssef@univ-ibntofail.ac.ma, \\ merouane.debbahasupelec.fr, aboutaj@fsr.ac.ma
}

\begin{abstract}
Energy efficiency is a foremost concern in Wireless Sensor Networks (WSNs). It aims to maximize the network lifetime which is defined as the time duration until the battery depletion of the first node. The aim of our approach is to provide the optimal transmission power taking into account the signal to noise ratio (SNR) constraint at the Fusion Center (FC) while guaranteeing the required performance. In this article, we address the lifetime maximization problem under non-orthogonal channels assuming two cases. In the first case, the nodes have the perfect knowledge of all channel gains. While in the second case, we propose several extensions to the unacknowledged channel gains by the nodes. In both cases, we consider that the nodes transmit their data to the FC over Quasi-Static Rayleigh fading Channel (QSRC). Simulation results show that the proposed optimal power allocation method maximizes the network lifetime better then the EP method.
\end{abstract}

Keywords: Energy-Efficiency, WSNs, MIMO Cooperative, Cooperation Communication, Optimal Power Allocation.

\section{Introduction}

Wireless Sensor Networks (WSNs) represent a technological revolution resulting from convergence of electronic and wireless communication systems. A WSN is a special network composed from a large number of nodes equipped with an embedded processor, sensors and a radio. These nodes have very limited resources which should be wisely used while trying to provide an acceptable QoS. Since nodes in WSNs are battery powered and changing batteries is a very difficult operation due to highly varying topology and deployment characteristics, the energy consumption should be taken into account in order to maximize nodes lifetime. Then, the most important objective for the WSN is to maximize the network lifetime by making the nodes run for a long time. The network lifetime has been defined in various ways. It may be defined as the time until the first sensor runs out of energy as in [1], others have defined it as the time until the last sensor runs out of energy[2][3]. In this work, we 
consider the first assumption. In literature, there are several works that have treated the same issue and which will be quoted in brief in this article.

We begin by mentioning the work of Belmega et al. in [4] which conclude that MIMO systems are more energy efficient than SISO systems if only the transmitted power consumption is taken into account. However, when the circuitry energy consumption is considered, this conclusion is no longer true.

In the WSN, however, the node cannot carry multiple antennas at the same time due to his limited physical size. Therefore, a new transmission technique called "Cooperative MIMO" has been proposed in [5] [6] for a better diversity reception. This technique is based on the cooperation principle where the participating nodes (relays) form a distributed antenna array to achieve the diversity gain of the MIMO system, in other words, the MIMO technology is virtually introduced.

Several studies have addressed the problem of maximizing the network lifetime using various methods for minimizing energy consumption. In [7] [8] optimal solutions are presented for maximizing a static network lifetime through a graph theoretic approach using static broadcast tree. In [8] Thomas et al. have presented an optimal solution for maximizing the network lifetime through a graph theoretic approach using a static multicast tree. Bhardwaj et al. [9], [10] have explored the fundamental limits of energy-efficient collaborative data-gathering by deriving upper bounds on the lifetime of increasingly sophisticated sensor networks assuming that sensor nodes only consume energy when they process, send or receive data. In [7], the authors have studied the node density vs. network lifetime tradeoff for a cell-based energy conservation technique.

In this paper, we introduce a novel method for maximizing the network lifetime under the non-orthogonal channels taking into account the total SNR constraint at the FC. The next part of this paper is organized as follows: Section 2 explains our method applied to the non-orthogonal channels considering that the nodes have direct access to the FC and they transmit their data over a QSRC. We assume two cases; in the first case, we consider that the nodes have the perfect knowledge of all channel gains. In the second, we consider that the nodes do not have knowledge of all channel gains. Section 3, presents the conducted experiments and the last section concludes the paper.

\section{Background and Definitions}

In this section, we give a background and precisely define the terms used throughout this paper. We assume a Fusion Centre (FC) and M sensors randomly distributed in the area of interest and these sensors have a direct access to the FC (see figure 1). We consider that the nodes transmit their data over quasi-static Rayleigh fading channels and each sensor has an initial energy noted by $E_{\text {int }}$.

We assume that the sensed observation, when a monitored event occurs, is contaminated with Additive White Gaussian Noise (AWGN) noted by $n_{i}$. The noisy observation from the $i^{\text {th }}$ sensor can be written as:

$$
x_{i}=\theta+n_{i}
$$


Where $\theta$ is the actual parameter being measured and $n_{i}$ is the additive complex Gaussian noise with $n_{i} \sim C \mathrm{~N}\left(0 ; \sigma_{i t}^{2}\right)$. To ensure that this noisy observation $x_{i}$ to be transmitted to the FC, it must be multiplied by the transmitter gain $w_{i}$. We note that the transmission power is written as $p_{i}=w_{i}^{2}\left(1+\sigma_{i t}^{2}\right)$ considering that $E\left[\theta^{2}\right]=1$, where, $E[:]$ is the mathematical expectation operator [14].

We assume that the noisy observations transmitted to the FC have another noise noted $n_{r}$ with an additive complex Gaussian distribution $n_{r} \sim C \mathrm{~N}\left(0 ; \sigma_{r}^{2}\right)$ and $h_{i}$ is the $i^{\text {th }}$ channel coefficient from the sensor $i$ to the FC. We consider that $\left|h_{i}\right|$ has a Rayleigh distribution where $\sigma_{h i}^{2}$ represents the well known variance, where,

$$
f\left(\left|h_{i}\right|\right)=\frac{\left|h_{i}\right| e^{\left(\frac{-\left|h_{i}\right|^{2}}{2 \sigma_{h i}^{2}}\right)}}{\sigma_{h i}^{2}}
$$

In this article, our goal is to maximize the network lifetime that is written as follows:

$$
L=N * T
$$

Where the $T$ is the period measurement of channel condition (we consider that $T=1$ to simplify), $N$ is the number of transmissions before the network misses energy. Consequently, to maximize the networks lifetime it is sufficient to maximize the number of transmission for each sensor, taking into account the estimation of overall SNR constraint at the FC, then the general formulation of our problem as:

$$
\left\{\begin{array}{c}
\operatorname{Max} E[N] \\
S N R \geq \gamma \\
0 \leq P_{i} \leq E_{\text {int }}
\end{array}\right.
$$

In our work, we focus on the optimal power allocation problem for WSNs under NonOrthogonal channels assuming two cases quoted previously. In addition, we suppose a linear minimum mean square-error (LMMSE) detector is used at the receiver.

\subsection{Non-orthogonal Channel (Known Channel States)}

We assume $M$ sensors randomly distributed in the area of interest using nonorthogonal channels between the FC and each sensor (Figure 1). We consider that the nodes have Channel State Information (CSI).

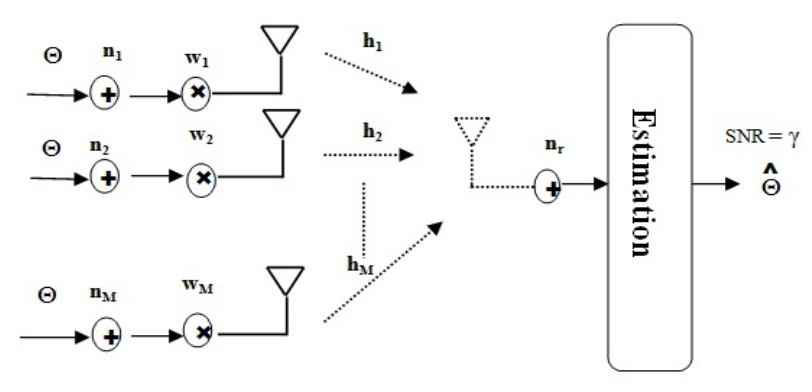

Fig. 1. System model 
The received signal at the $\mathrm{FC}$ is defined by:

$$
y=\sum_{i=1}^{M} h_{i} w_{i}\left(\theta+n_{i}\right)+n_{r}
$$

Assuming that we use real channels, the SNR at the FC corresponding to $\mathrm{M}$ sensors using the MMSE detector is given by:

$$
S N R=\frac{\left(\sum_{i=1}^{M}\left|h_{i}\right| w_{i}\right)^{2}}{\sum_{i=1}^{M} w_{i}^{2}\left|h_{i}\right|^{2} \sigma_{i t}^{2}+\sigma_{r}^{2}}
$$

Our aim is to maximize the batteries lifetime duration while keeping the expected value of SNR greater than or equal to a target value $\gamma$.

At the $l^{\text {th }}$ instant, maximizing the lifetime relies on minimizing the power consumption; therefore, the problem formulation is given as follows:

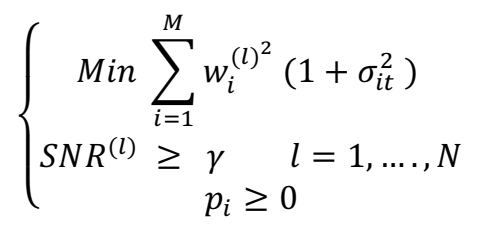

To find the optimal points, we use the Lagrange method while satisfying the constraints quoted before. The Lagrangian $£$ can be written as follows:

$$
\begin{aligned}
\mathcal{L}\left(\mathrm{w}_{\mathrm{i}}^{(\mathrm{l})}, \lambda, v\right) & =\sum_{\mathrm{i}=1}^{\mathrm{M}} \mathrm{w}_{\mathrm{i}}^{(\mathrm{l})^{2}}\left(1+\sigma_{\mathrm{it}}^{2}\right)-\sum_{\mathrm{i}=1}^{\mathrm{M}} \lambda_{\mathrm{i}} \mathrm{w}_{\mathrm{i}}^{(\mathrm{l})} \\
+v_{\mathrm{l}} & {\left[\gamma\left[\sum_{\mathrm{i}=1}^{\mathrm{M}} \mathrm{w}_{\mathrm{i}}^{(\mathrm{l})^{2}}\left|\mathrm{~h}_{\mathrm{i}}^{(\mathrm{l})}\right|^{2} \sigma_{\mathrm{it}}^{2}+\sigma_{\mathrm{r}}^{2}\right]-\left[\sum_{\mathrm{i}=1}^{\mathrm{M}} \mathrm{w}_{\mathrm{i}}^{(\mathrm{l})}\left|\mathrm{h}_{\mathrm{i}}^{(\mathrm{l})}\right|\right]^{2}\right] }
\end{aligned}
$$

Let consider the Karush-Kuhn-Tucker (KKT) [14] conditions for the problem:

$$
\left\{\begin{array}{c}
\lambda_{\mathrm{i}} \geq 0, \mathrm{v}_{\mathrm{l}} \geq 0, \quad \lambda_{\mathrm{i}} \mathrm{w}_{\mathrm{i}}^{(\mathrm{l})}=0 \forall \mathrm{l}, \mathrm{i}=1,2 \\
v_{\mathrm{l}}\left[\gamma\left[\sum_{\mathrm{i}=1}^{\mathrm{M}} \mathrm{w}_{\mathrm{i}}^{(\mathrm{l})}\left|\mathrm{h}_{\mathrm{i}}^{(\mathrm{l})}\right|^{2} \sigma_{\mathrm{it}}^{2}+\sigma_{\mathrm{r}}^{2}\right]-\left[\sum_{\mathrm{i}=1}^{\mathrm{M}} \mathrm{w}_{\mathrm{i}}^{(\mathrm{l})}\left|\mathrm{h}_{\mathrm{i}}^{(\mathrm{l})}\right|\right]^{2}\right]=0 \\
\frac{\partial \mathcal{L}}{\partial \mathrm{w}_{\mathrm{k}}^{(\mathrm{l})}}=0
\end{array}\right.
$$

Then, the partial derivative of $£$ with respect to $\mathrm{w}_{\mathrm{k}}$ is:

$$
\frac{\partial \mathcal{L}}{\partial \mathrm{w}_{\mathrm{k}}^{(\mathrm{l})}}=2 \mathrm{w}_{\mathrm{k}}^{(\mathrm{l})}\left(1+\sigma_{\mathrm{kt}}^{2}\right)-\lambda_{\mathrm{k}}+2 v_{\mathrm{l}} \gamma\left|\mathrm{h}_{\mathrm{k}}^{(\mathrm{l})}\right|^{2} \sigma_{\mathrm{kt}}^{2} \mathrm{w}_{\mathrm{k}}^{(\mathrm{l})}-2 v_{\mathrm{l}}\left|\mathrm{h}_{\mathrm{k}}^{(\mathrm{l})}\right|\left[\sum_{\mathrm{i}=1}^{\mathrm{M}} \mathrm{w}_{\mathrm{i}}^{(\mathrm{l})}\left|\mathrm{h}_{\mathrm{i}}^{(\mathrm{l})}\right|\right]
$$


Taking into account the KKT conditions, we find that $v_{\mathrm{l}}>0$ and $\lambda_{\mathrm{k}}=0$. Thus,

$$
\mathrm{w}_{\mathrm{k}}^{(\mathrm{l})}=\frac{v_{\mathrm{l}}\left|\mathrm{h}_{\mathrm{k}}^{(\mathrm{l})}\right|\left(\sum_{\mathrm{i}=1}^{\mathrm{M}} \mathrm{w}_{\mathrm{i}}^{(\mathrm{l})}\left|\mathrm{h}_{\mathrm{i}}^{(\mathrm{l})}\right|\right)}{\left(1+\sigma_{\mathrm{kt}}^{2}\right)+v_{\mathrm{l}} \gamma\left|\mathrm{h}_{\mathrm{k}}^{(\mathrm{l})}\right|^{2} \sigma_{\mathrm{kt}}^{2}}
$$

To find the value of $\sum_{\mathrm{i}=1}^{\mathrm{M}} \mathrm{w}_{\mathrm{i}}^{(1)}\left|\mathrm{h}_{\mathrm{i}}^{(1)}\right|$ we replace (8) in (7), and it becomes:

$$
\left[\sum_{\mathrm{i}=1}^{\mathrm{M}} \mathrm{w}_{\mathrm{i}}^{(\mathrm{l})}\left|\mathrm{h}_{\mathrm{i}}^{(\mathrm{l})}\right|\right]^{2}=\frac{\gamma \sigma_{\mathrm{r}}^{2}}{1-\gamma v_{\mathrm{l}}^{2}\left[\sum_{\mathrm{i}=1}^{\mathrm{M}}\left[\frac{\left|\mathrm{h}_{\mathrm{i}}^{(\mathrm{l})}\right|^{4}}{\left[\left(1+\sigma_{\mathrm{it}}^{2}\right)+v_{\mathrm{l}} \gamma\left|\mathrm{h}_{\mathrm{i}}^{(\mathrm{l})}\right|^{2} \sigma_{\mathrm{it}}^{2}\right]^{2}} \sigma_{\mathrm{it}}^{2}\right]\right.}
$$

Finally, equation (8) becomes:

$$
\mathrm{w}_{\mathrm{k}}^{(\mathrm{l})}=\frac{v_{\mathrm{l}}\left|\mathrm{h}_{\mathrm{k}}^{(\mathrm{l})}\right| \sigma_{\mathrm{r}} \sqrt{\gamma}}{\left[\left(1+\sigma_{\mathrm{kt}}^{2}\right)+v_{\mathrm{l}} \gamma\left|\mathrm{h}_{\mathrm{k}}^{(\mathrm{l})}\right|^{2} \sigma_{\mathrm{kt}}^{2}\right]\left[1-\gamma v_{\mathrm{l}}^{2}\left[\sum_{\mathrm{i}=1}^{\mathrm{M}} \frac{\left|\mathrm{h}_{\mathrm{i}}^{(\mathrm{l})}\right|^{4}}{\left[\left(1+\sigma_{\mathrm{it}}^{2}\right)+v_{\mathrm{l}} \gamma\left|\mathrm{h}_{\mathrm{i}}^{(\mathrm{l})}\right|^{2} \sigma_{\mathrm{it}}^{2}\right]^{2}} \sigma_{\mathrm{it}}^{2}\right]\right.}
$$

Now, the challenge is to find the value of $v_{1}$.Therefore, we multiply equation (8) by $\left|h_{k}^{(l)}\right|$, After that, we compute the sum of all the resulting equations, we obtain:

$$
\sum_{\mathrm{i}=1}^{\mathrm{M}}\left|\mathrm{h}_{\mathrm{k}}^{(\mathrm{l})}\right| \mathrm{w}_{\mathrm{k}}^{(\mathrm{l})}\left[1-\sum_{\mathrm{i}=1}^{\mathrm{M}} \frac{v_{\mathrm{l}}\left|\mathrm{h}_{\mathrm{k}}^{(\mathrm{l})}\right|^{2}}{\left(1+\sigma_{\mathrm{kt}}^{2}\right)+v_{\mathrm{l}} \gamma\left|\mathrm{h}_{\mathrm{k}}^{(\mathrm{l})}\right|^{2} \sigma_{\mathrm{kt}}^{2}}\right]=0
$$

Since $\sum_{\mathrm{i}=1}^{\mathrm{M}} \mathrm{h}_{\mathrm{k}} \mathrm{w}_{\mathrm{k}}^{(\mathrm{l})} \neq 0$, we obtain:

$$
\sum_{\mathrm{i}=1}^{\mathrm{M}} \frac{\left|\mathrm{h}_{\mathrm{k}}^{(\mathrm{l})}\right|^{2}}{1+\sigma_{\mathrm{kt}}^{2}\left[1+v_{\mathrm{l}} \gamma\left|\mathrm{h}_{\mathrm{k}}^{(\mathrm{l})}\right|^{2}\right]}=\frac{1}{v_{\mathrm{l}}}
$$

This equation is not written in a closed-form solution. So, it can be solved numerically using the function "Fminsearch" [13].

\subsection{Non-orthogonal Channel (Unknown Channel States)}

Since the previous assumption is not actually valid for some practical systems, then, in this section, we consider the same assumptions of the previous section except that in which the nodes do not have a Channel State Information (CSI). The received signal at the FC from $i^{\text {th }}$ sensor is defined by: 


$$
y_{i}=h_{i} w_{i}\left(\theta+n_{i}\right)+n_{r}
$$

The average SNR at the FC in this case is not similar to that in the previous section, it can be written as follows:

$$
S N R=\frac{\sum_{i=1}^{M} w_{i}^{2}\left|h_{i}^{(l)}\right|^{2}}{\sum_{i=1}^{M} \sigma_{i t}^{2} w_{i}^{2}\left|h_{i}^{(l)}\right|^{2}+\sigma_{r}^{2}}
$$

Our aim is to maximize the lifetime of our network by taking into account the estimation of overall SNR at FC. To maximize the networks lifetime it is adequate to minimize the transmission power for each sensor, then, our problem formulation as:

$$
\left\{\begin{array}{c}
\operatorname{Min} \sum_{i=1}^{M} w_{i}^{(l)^{2}}\left(1+\sigma_{i t}^{2}\right) \\
S N R \geq \gamma \\
P_{i} \geq 0
\end{array}\right.
$$

To find the optimum power, we will use the Lagrange method as an optimization method, while satisfying the constraints quoted before. Using the equation (12), the Lagrangian $€$ can be written as follows:

$$
\begin{aligned}
\mathcal{L}\left(w_{i}^{(l)}, \lambda, v\right)= & \sum_{i=1}^{M} w_{i}^{(l)^{2}}\left(1+\sigma_{i t}^{2}\right)-\sum_{i=1}^{M} \lambda_{i} w_{i}^{(l)^{2}} \\
& -v\left[\sum_{i=1}^{M} w_{i}^{2}\left|h_{i}^{(l)}\right|^{2}-\gamma \sum_{i=1}^{M} \sigma_{i t}^{2} w_{i}^{2}\left|h_{i}^{(l)}\right|^{2}-\gamma \sigma_{r}^{2}\right]
\end{aligned}
$$

With the Karush-Kuhn-Tucker (KKT) Conditions for the problem are given by:

$$
\left\{\begin{array}{c}
\lambda_{i} \geq 0, v \geq 0, \lambda_{i} w_{i}^{(l)}=0 \\
{\left[\sum_{i=1}^{M} w_{i}^{2}\left|h_{i}^{(l)}\right|^{2}-\gamma \sum_{i=1}^{M} \sigma_{i t}^{2} w_{i}^{2}\left|h_{i}^{(l)}\right|^{2}-\gamma \sigma_{r}^{2}\right]=0} \\
\frac{\partial \mathcal{L}}{\partial w_{k}^{(l)}}=0
\end{array}\right.
$$

The partial derivative of $\mathcal{L}$ with respect to $p_{k}^{(l)}$ is:

$$
\frac{\partial \mathcal{L}}{\partial w_{k}^{(l)}}=1+\sigma_{k t}^{2}-\lambda_{k}-v\left|h_{k}^{(l)}\right|^{2}\left[1-\gamma \sigma_{k t}^{2}\right]=0
$$


In that case, the Lagrangian method does not lead us to solve our problem. According to the equation (12), and knowing that the denominator is positive, we have:

Then,

$$
\sum_{i=1}^{M} w_{i}^{2}\left|h_{i}^{(l)}\right|^{2}-\gamma \sum_{i=1}^{M} \sigma_{i t}^{2} w_{i}^{2}\left|h_{i}^{(l)}\right|^{2} \geq \gamma \sigma_{r}^{2}
$$

$$
\sum_{i=1}^{M} w_{i}^{2}\left|h_{i}^{(l)}\right|^{2}\left(1-\gamma \sigma_{i t}^{2}\right) \geq \gamma \sigma_{r}^{2}
$$

We can observe that our equation is written as: $a^{T} X \geq b$ that is called a linear matrix inequality (LMI) [17] in $\mathrm{x}: \quad a^{T} X=x_{1} a_{1}+\ldots+x_{n} a_{n} \geq b$, with $X=\left[w_{1}^{2}, w_{2}^{2}, \ldots ., w_{M}^{2}\right], \quad b=\gamma \sigma_{r}^{2} \quad$ and $\quad \mathrm{a}=\left[\left|h_{1}^{(l)}\right|^{2}\left(1-\sigma_{1 t}^{2}\right),\left|h_{2}^{(l)}\right|^{2}(1-\right.$ $\left.\left.\sigma_{2 t}^{2}\right), \ldots \ldots .\left|h_{M}^{(l)}\right|^{2}\left(1-\sigma_{M t}^{2}\right)\right]$. Then, the problem can be solved numerically.

\section{Simulation}

Several simulations have been conducted using MATLAB in order to compare and evaluate the behavior of both the Equal Power (EP) method [16] and our novel approach. For each simulation, we study the network lifetime while increasing the number of nodes. The simulations parameters are generated randomly such that each parameter $\mathrm{p}$ belongs to a uniform distribution between $\psi$ and $\varphi, p \in U[\psi ; \varphi]$.

\subsection{Non-orthogonal Channel (Known Channel States)}

Figure 2 shows the lifetime network while increasing the number of nodes using nonorthogonal channels where the channel coefficients are known. As it can be seen, the proposed approach improves EP method concerning the network lifetime. Actually, the network lifetime is extended by an average of 82, 80\%. Table 1 shows the parameters used for simulations.

Table 1. Simulations parameters

\begin{tabular}{c|l}
\hline Estimate & Parameters \\
\hline $\mathrm{U}[0.1,0.2]$ & $\sigma_{\mathrm{hi}}^{2}:$ The variances of channel estimation \\
\hline 0.5 & $\sigma_{\mathrm{r}}^{2}:$ The noise variance at the FC \\
\hline $\mathrm{U}[0.02,0.1]$ & $\sigma_{\mathrm{it}}^{2}:$ The observation noise variances \\
\hline $\mathrm{U}[200,500]$ & $\varepsilon_{\mathrm{i}}:$ The initial energy \\
\hline
\end{tabular}

\subsection{Non-orthogonal Channel (Unknown Channel States)}

In Figure 3, we can observe that our new method is more effective than the EP method concerning network lifetime. The batteries lifetime duration is extended by an average of $79,98 \%$. Table 1 shows the parameters used for simulations. 


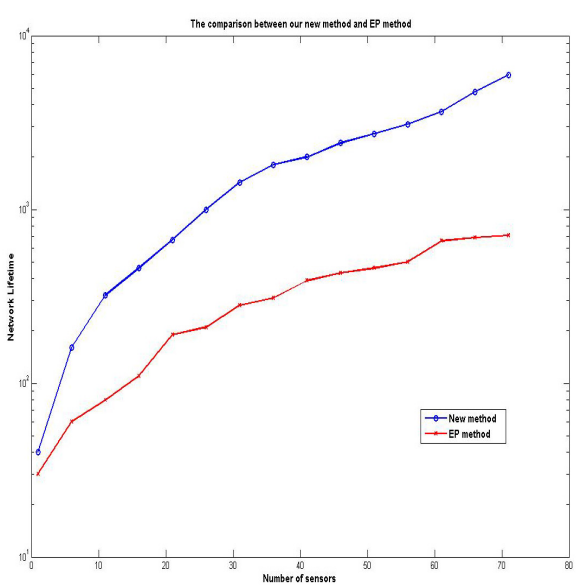

Fig. 2. Non-Orthogonal Channel (Known Channel States)

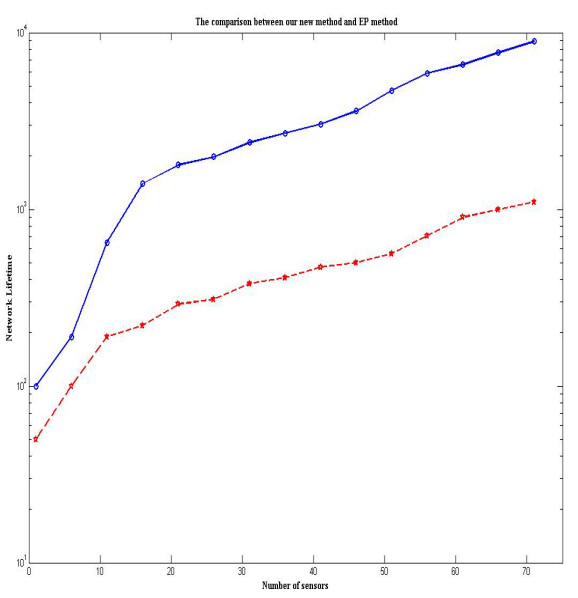

Fig. 3. Non-Orthogonal Channel (Unknown Channel States)

\section{Conclusion}

This paper presented a new algorithm which aims to maximize the network lifetime under Non-Orthogonal channel configuration. This method takes into consideration the estimation of the overall SNR at the FC. We showed that our new method in the both cases consumes less energy than the EP method. The future work is to adapt our new method to the wireless communication using energy harvesting transmitters.

\section{References}

1. Ratnasamy, S., Estrin, D., Govindan, R., Karp, B., Yin, L., Shenker, S., Yu, F.: Datacentric storage in sensornets. In: Proceeding of the ACM SIGCOMM Computer Communication Review, Pittsburgh, PA, USA, pp. 137-142 (2002)

2. Choi, W., Shah, P., Das, S.K.: A Framework for Energy-Saving Data Gathering Using Two-Phase Clustering in Wireless Sensor Networks. In: Proceeding of the Mobile and Ubiquitous Systems, Boston, pp. 203-212 (2004)

3. Ephremides, A.: Energy concerns in wireless networks. IEEE Wireless Communications 9(4) (2002)

4. Belmega, E.V., Lasaulce, S., Debbah, M.: A survey on energy-efficient communications. In: IEEE Intl. Symp. on Personal, Indoor and Mobile Radio Communications (PIMRC 2010), Istanbul, Turkey, p. 289 (2010) (invited paper)

5. Cui, S., Goldsmith, A.J., Bahai, A.: Energy efficiency of MIMO and cooperative MIMO techniques in sensor networks. IEEE Jour. on Selected Areas in Comm. 22(6), 1089-1098 (2004)

6. Nguyen, T., Berder, O., Sentieys, O.: Cooperative MIMO schemes optimal selection for wireless sensor networks. In: IEEE 65th Vehicular Technology Conference, pp. 85-89 (2007) 
7. Winters, J.: The diversity gain of transmit diversity in wireless systems with Rayleigh fading. IEEE Transactions on Vehicular Technology 47(1), 119-123 (1998)

8. Thomas Hou, Y., Shi, Y., Hanif Sherali, D., Jeffrey Wieselthier, E.: Multicast Communications in Ad HocvNetworks Using Directional Antennas: A Lifetime-Centric Approach. IEEE Transactions on Vehicular Technology 56(3) (2007)

9. Chandrakasan, A.: Design considerations for distributed micro-sensor systems. In: Custom Integrated Circuits Conference (CICC), pp. 279-286 (1999)

10. Shih, E.: Physical layer driven protocol and algorithm design for energy-efficient wireless sensor networks. In: Proc. of the Seventh Annual ACM/IEEE International Conference on Mobile Computing and Networking, pp. 272-286 (2001)

11. Bian, F., Goel, A., Raghavendra, C.S., Li, X.: Energy-efficient broadcasting in wireless ad hoc networks: lower bounds and algorithms. JINet 3, 149-166 (2002)

12. Wei, H., Sasaki, H., Kubokawa, J.: A decoupled solution of hydro-thermal optimal power flow problem by means of interior point method and network programming. IEEE Transactions on Power Systems 13(2), 286-293 (1998)

13. http://www.mathworks.com/help/techdoc/ref/ fminsearch.htmlFmin

14. Namin, F., Nosratinia, A.: Progmatic Lifetime maximization of cooperative Sensor Networks via a decomposition approoch. In: Acoustics, Speech and Signal Processing- Las Vegas, Nevada, U.S.A., vol. (4), p. 3017 (2008)

15. Boyd, S., Vandenberghe, L.: Convex Optimization. Cambridge University Press (2004)

16. Goudarzi, H., Pakravan, M.R.: Equal Power Allocation scheme for cooperative diversity. In: 4th IEEE/IFIP International Conference, Tashkent-uzbekistan, pp. 1-5 (2008) 